\title{
A Photochromic Bioconjugate with Photoactivatable Fluorescence for Superresolution Imaging
}

Janet Cusido, ${ }^{\dagger}$ Sherif Shaban Ragab, ${ }^{\dagger,}$ Ek Raj Thapaliya, ${ }^{\dagger}$ Subramani Swaminathan ${ }^{\dagger}$ Jaume Garcia-Amorós, ${ }^{\dagger}$ M. Julia Roberti, ${ }^{\S, \#}$ Beatriz Araoz, ${ }^{\S}$

\author{
Mercedes M. A. Mazza, ${ }^{\dagger}$ Shiori Yamazaki,${ }^{\dagger}$ Amy M. Scott ${ }^{\dagger}$ \\ Françisco M. Raymo*,† and Mariano L. Bossi ${ }^{*}{ }^{*}$
}

Laboratory for Molecular Photonics, Department of Chemistry, University of Miami, 1301 Memorial

Drive, Coral Gables, Florida, 33146-0431, Department of Photochemistry, Chemical Industries

Research Division, National Research Centre (NRC), El behouth Street, Dokki, Giza, 12622, Egypt and

INQUIMAE, Facultad de Ciencias Exactas y Naturales, Universidad de Buenos Aires, Pabellón 2,

Ciudad Universitaria, 1428 - Buenos Aires, Argentina

- Fitting Parameters for the Kinetic Traces of 10a upon Photoexcitation

- Absorption Spectra of 10a Recorded Before and After Multiple Excitation Cycles

- Events and Photons Histograms from Single-Molecule Localization Microscopy Images

$\dagger$ University of Miami

* National Research Centre

$\S$ Conicet - Universidad de Buenos Aires

\# Present address: EMBL, Heidelberg, Germany

* Corresponding Authors

FMR: E-Mail: fraymo@miami.edu; Tel.: +1 3052842639

MLB: E-Mail: mariano@qi.fcen.uba.ar; Tel.: +54 1145763380 
Table S1. Lifetimes $\left(\tau_{1}-\tau_{4}\right)$ extracted from the multiexponential fittings of the absorbance evolutions observed upon excitation of 10a with the corresponding relative contributions [a].

\begin{tabular}{|c|c|c|c|c|c|c|c|c|}
\hline 445 & $3.1 \times 10^{-3} \pm 4.4 \times 10^{-4}$ & 48 & $1.0 \times 10^{-1} \pm 1.0 \times 10^{-2}$ & 21 & $6.4 \pm 1.0$ & 31 & - & - \\
\hline 537 & $4.9 \times 10^{-4} \pm 1.6 \times 10^{-4}$ & 61 & $1.9 \times 10^{-2} \pm 2.9 \times 10^{-3}$ & 18 & $2.4 \times 10^{-1} \pm 6.9 \times 10^{-2}$ & 10 & $4.9 \pm 1.3$ & 11 \\
\hline
\end{tabular}

[a] The absorbance profiles (B-D in Figure 6) were recorded at the wavelength $(\lambda)$ listed upon photoexcitation ( $400 \mathrm{~nm}, 8 \mu \mathrm{J} / \mathrm{pulse})$ of a PBS solution (25 $\left.{ }^{\circ} \mathrm{C}\right)$ of 10a $\left(\sim 0.2 \mathrm{mg} \mathrm{mL}^{-1} \mathrm{mM}\right)$.

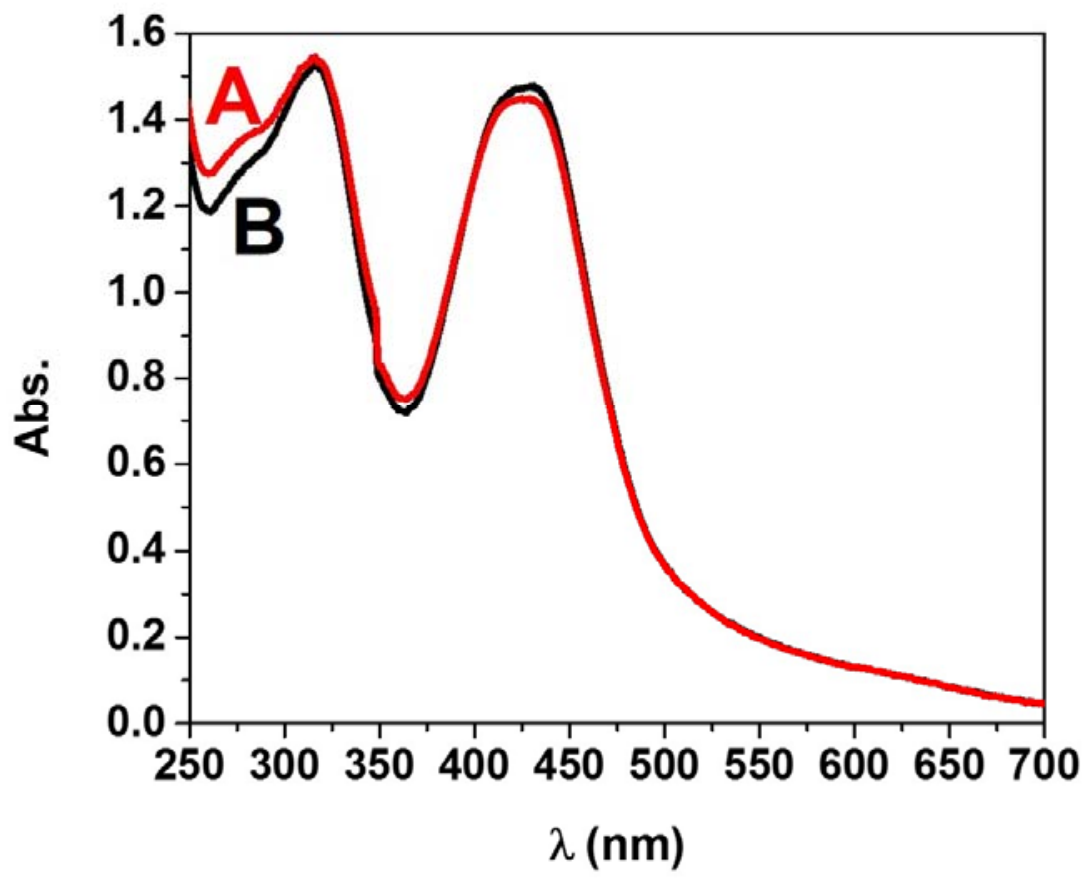

Figure S1. Absorption spectra of a PBS solution $\left(25^{\circ} \mathrm{C}\right)$ of 10a $\left(\sim 0.2 \mathrm{mg} \mathrm{mL}^{-1} \mathrm{mM}\right)$ recorded before (A) and after (B) 3 hours of photoexcitation $(400 \mathrm{~nm}, 8 \mu \mathrm{J} /$ pulse, $500 \mathrm{~Hz}$, IRF $70 \mathrm{fs})$. 

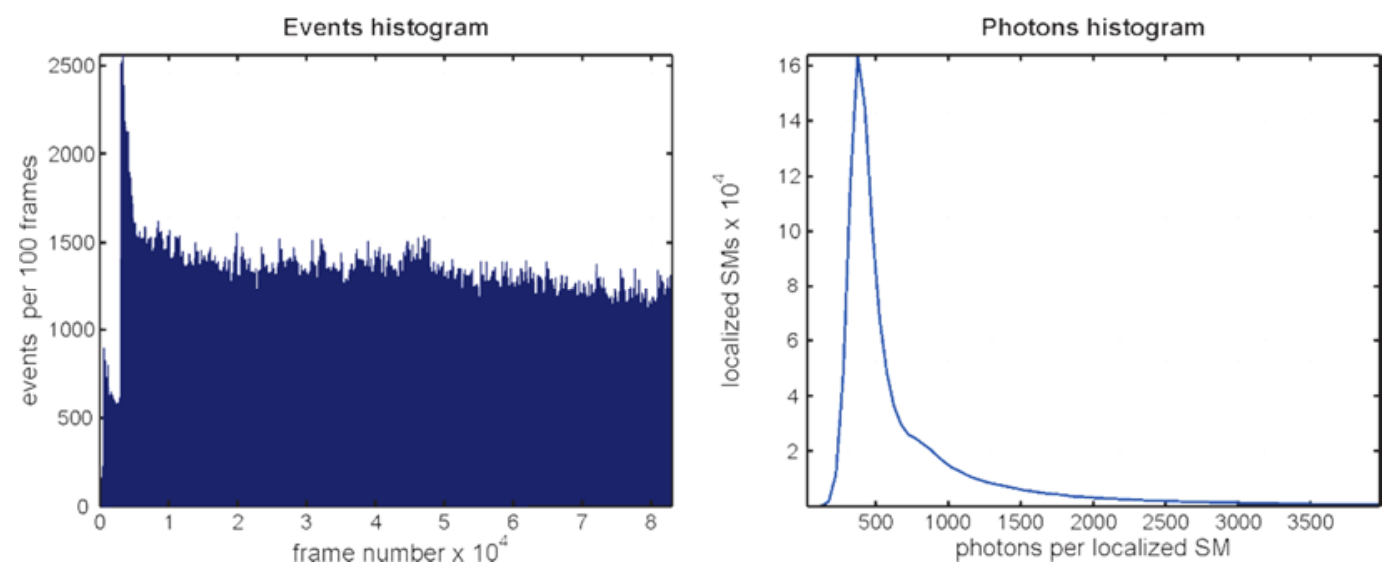

Figure S2. Left (Events Histogram): localized events per 100 frames, during the acquisition of the image in Figure 9 (main text). The two spikes observed at frame $\sim 500$ and at frame $\sim 3,000$ correspond to adjustments (increasing) of the activation laser $(405 \mathrm{~nm})$. This laser was again slightly increased at frame $\sim 22,000$ and at frame $\sim 41,000$. Right (Photons Histogram) number of photons detected per localized SM used to generate the superresolution image. The expected average number of photons is $\sim 600$. 